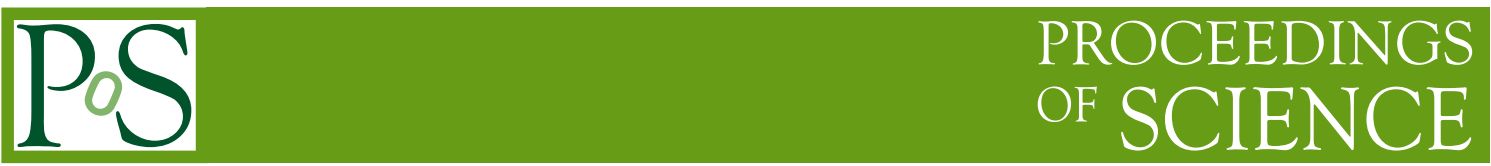

\title{
4D particle tracking with Resistive AC-Coupled Silicon Detectors (RSD)
}

\author{
Marco Mandurrino*† \\ INFN, Sezione di Torino \\ Via P. Giuria, 1 - 10125 Torino - Italy \\ E-mail: marco.mandurrinoato.infn.it
}

\begin{abstract}
We present the advantages of performing 4D particle tracking with Resistive AC-Coupled Silicon Detectors (RSD), a new paradigm in Silicon detectors with moderate internal multiplication. Their design is an evolution of the standard LGAD (Low-Gain Avalanche Diode) technology, and is based on the combination of a resistive $n$-implant, freezing the multiplied charges, and a capacitive oxide layer, coupling the signal with the readout pads. Having a homogeneous gain layer throughout the detector, the spatial granularity is realized through the segmentation of pads, while the timing information directly benefits from the good performances given by the internal multiplication. Such scheme will allow to completely eliminate the signal loss between active areas (or pixels) proper of LGAD-based silicon trackers and to fully exploit the potentialities of high-luminosity scenarios foreseen in near-future colliders, thanks to their intrinsic $100 \%$ fill-factor (the ratio between the active and the total area). Here we present both the design and a set of laboratory characterizations of the first production run RSD1 by the Fondazione Bruno Kessler (FBK) in Trento.
\end{abstract}

European Physical Society Conference on High Energy Physics - EPS-HEP2019 -

10-17 July, 2019

Ghent, Belgium

\footnotetext{
*Speaker.

${ }^{\dagger}$ on behalf of the RSD group.
} 


\section{Introduction}

The 4D-tracking with Silicon sensors is the enabling solution for particle detection in near-future HEP experiments [1,2], where high-luminosity environments require high-resolution particle tagging both in space and in time with the same detector. To this aim, in the past years, scientists working on such R\&D focused their efforts on the development of several technologies allowing the determination of the particle time of arrival with a precise resolution (say, few tens of picoseconds) along with the best spatial reconstruction as possible. One of the most promising is the LGAD (Low-Gain Avalanche Diode) technology, which exploits the inclusion of an additional $p^{+}$ implant just below the $n$-cathode of a $p$-type Silicon sensor [3]. Biasing the diode in reverse polarization above a threshold voltage, the $p^{+}$layer (or gain layer) experiences an electric field large enough $(\sim 300 \mathrm{kV} / \mathrm{cm})$ to produce the charge avalanche multiplication mechanism. In this regime, for each primary charge produced through impact ionization by a charged particle crossing the device we obtain a certain number of secondary electron/hole pairs. Such number represents the multiplication factor (or gain, $G$ ) of the detector, which is a function of the applied reverse bias (or, in other words, of the field within the gain layer).

Having an internal gain is beneficial for timing applications because it mainly provides signal multiplication, while the readout amplification would enhance also the electronic noise. Indeed, when the gain is moderate, the $\mathrm{S} / \mathrm{N}$ (signal to noise) ratio is high and, if the gain increases, the slew rate becomes higher as well. Being the $\mathrm{S} / \mathrm{N}$ and the slew rate directly related to the time resolution $\sigma_{t}$ [4], several simulations and measurements demonstrated that, detecting high-energy charged particles, a gain $G \sim 10-20$ in thin sensors $\left(\sim 50 \mu \mathrm{m}\right.$ ) allows to reach $\sigma_{t} \sim 30 \mathrm{ps}$ [5]. Another key aspect characterizing the LGAD technology is its radiation tolerance: as it has been already proven on UFSD (Ultra-Fast Silicon Detectors, i.e. LGAD-based detectors optimized for timing) this technology can operate in radiation-intense environments, with fluence up to $\phi \sim 10^{15} \mathrm{n}_{\mathrm{eq}} / \mathrm{cm}^{2}$ [6], a value which is fully compatible with the expectations for the MIP Endcap Timing Layer (ETL) in the High-Luminosity upgrade of CMS at CERN.

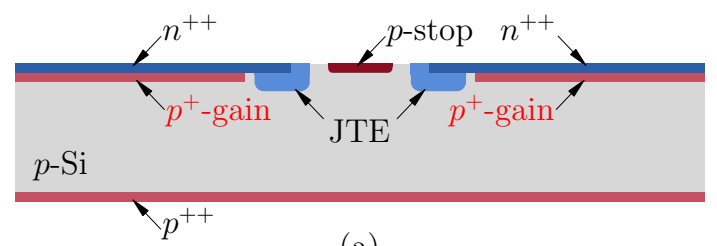

(a)

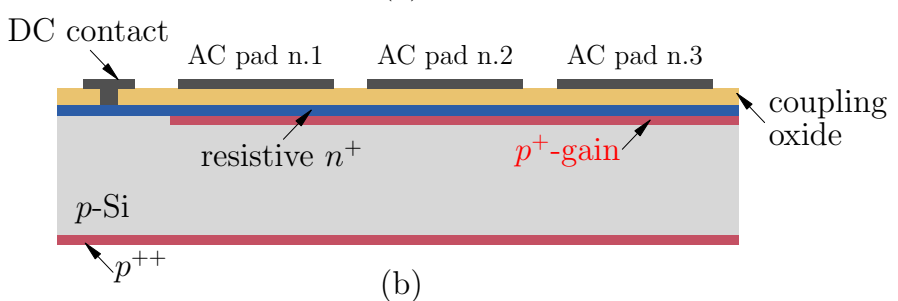

(b)

Figure 1: Cross-section of (a) two adjacent active areas in an LGAD-based detector, showing the JTE (Junction Termination Extension) and the $p$-stop segmentation implants, and (b) of an RSD device.

When in operation, the active areas of a typical segmented LGAD-based detector - represented by each gain implant - produces very-high electric fields. In order to prevent short-circuit between 
pixels, to well confine the field lines and, thus, to avoid early breakdown at the edges, some additional segmentation implants are usually adopted (see panel (a) of figure 1). In correspondence of such structures the charges are no more multiplied and, for this reason, the segmentation implants reduce the ratio between the active and the total detector area (called fill-factor). Contemporarily, the most important experiments working in high-luminosity conditions require a detector coverage of at least $95 \%[2,7]$. To meet all this requirements with the goal of developing particle detectors having high spatial resolution while maintaining the optimal properties of the LGAD technology in terms of timing, we simulated, designed, produced and tested a new generation of particle trackers called RSD (Resistive AC-Coupled Silicon Detectors), i.e. LGAD devices based on the resistive AC-coupled readout paradigm (see panel (b) of figure 1).

RSD are characterized by a continuous gain layer, without any segmentation. Therefore, the spatial information of tracks is reconstructed through two main key elements: a $n^{+}$layer and a coupling oxide. The first one works as a resistive sheet, freezing the multiplied charges for a characteristic time which has to be long enough for the signal to be read but sufficiently short to minimize the pile-up effect. The second feature, instead, allows to induce a signal on the metal pads through the capacitive coupling [8]. The RC constant of the readout equivalent circuit determines the waveform shape and - in particular - the discharge time, which is essential to obtain a good timing performance.

\section{RSD devices design}

To determine the optimum choice of the physical and technological parameters involved in the RSD signal formation, both Spice and 3D TCAD (Technology Computer-Aided Design) simulations have been carried out. The simple circuital modeling provided their approximative range,
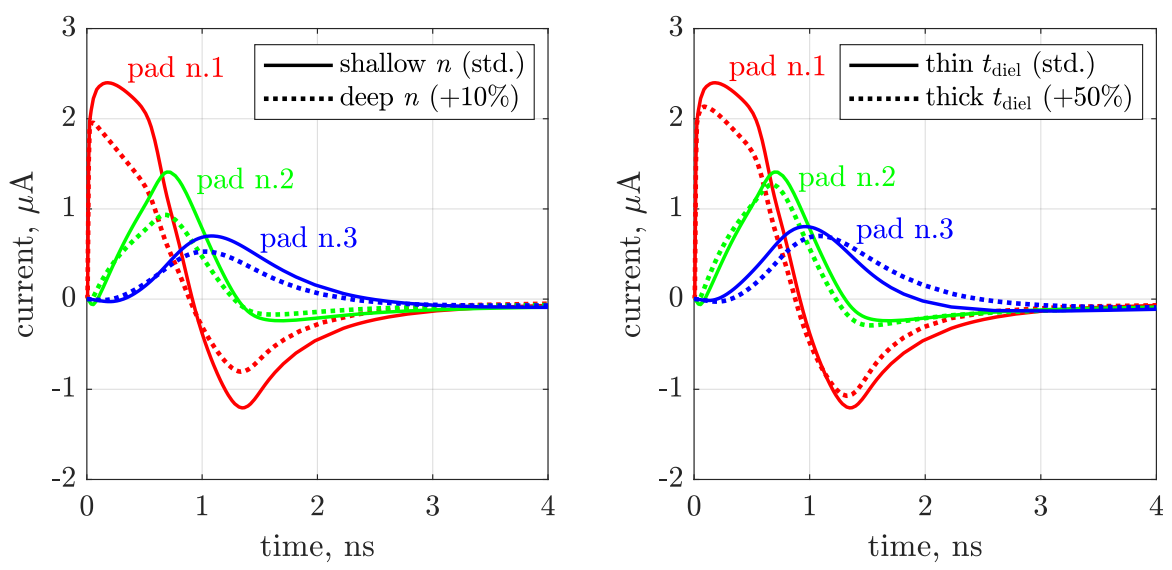

Figure 2: RSD signals simulated with a TCAD tool for a $50-\mu \mathrm{m}$-pitch $1 \times 3$ pad module, with active thickness $55 \mu \mathrm{m}$ and $V_{\text {bias }}=300 \mathrm{~V}$, for a MIP heavy ion passing perpendicularly to pad n.1. The waveforms are calculated by varying the $n$-cathode depth (left) or the dielectric thickness (right) with respect to the foundry's standard values. 
while multi-parametric numerical simulations allowed to determine the correct implantation profiles and to optimize the detector geometry based on its signals and on some microscopic quantities, such as the internal electric field. As an example, Figure 2 shows two different physics-based TCAD simulations carried out to investigate the coupling between the charges multiplied in the gain layer and the readout by tuning the $n$-cathode depth (left plot) and the dielectric thickness (right plot). In all calculations, a 55- $\mu$ m-thick $1 \times 3$ RSD structure with $50-\mu \mathrm{m}$-pitch and $V_{\text {bias }}=300 \mathrm{~V}$ has been accounted for, while the signals are obtained by simulating the injection of a MIP (minimum ionizing particle) heavy ion on pad $\mathrm{n} .1$ and acquiring the signals induced on all the three pads (see panel (b) of figure 1). As one may see, using a deep cathode or a thick dielectric layer (dashed lines) produces lower signals with respect to the standard fabrication rules (solid lines).

After an intense simulating campaign, we submitted in early 2019 a set of layout masks for a first batch of detectors - called RSD1 - to the foundry FBK (Fondazione Bruno Kessler), in Trento. The production, released in May 2019, included both epitaxial (Epi) and Float Zone (FZ) $6^{\prime \prime}$ Silicon wafers with an active thickness of $55 \mu \mathrm{m}$, that have been processed by FBK through the step-and-repeat (or stepper) lithography. Due to the high number of parameters involved in the RSD paradigm, 15 wafers have been used in this run, in order to explore a sufficiently wide range of values for what concerns the most important implants and features (see the table in figure 3). In particular, the $n^{+}$dose is expressed by a progressive letter (A, B and C), proportional to the implantation energy, the $p^{+}$(gain) dose, instead, is in terms of the same splits used in the UFSD productions (see [6]) and, finally, the dielectric has the two configurations low (L) or high (H), obtained by slightly changing the growth thickness during the deposition process.

\begin{tabular}{|c|c|c|c|c|}
\hline Wafer \# & type & $n^{+}$dose & $p^{+}$dose & diel. thickness \\
\hline 1 & FZ & A & 0.92 & $\mathrm{~L}$ \\
2 & FZ & A & 0.94 & $\mathrm{~L}$ \\
3 & Epi & A & 0.94 & $\mathrm{~L}$ \\
4 & FZ & A & 0.94 & H \\
5 & FZ & A & 0.96 & $\mathrm{~L}$ \\
6 & Epi & B & 0.92 & $\mathrm{~L}$ \\
7 & FZ & B & 0.94 & $\mathrm{~L}$ \\
8 & FZ & B & 0.94 & $\mathrm{~L}$ \\
9 & FZ & B & 0.96 & $\mathrm{~L}$ \\
10 & FZ & B & 0.96 & H \\
11 & FZ & C & 0.92 & L \\
12 & Epi & C & 0.94 & $\mathrm{~L}$ \\
13 & FZ & C & 0.94 & $\mathrm{~L}$ \\
14 & Epi & C & 0.96 & H \\
15 & FZ & C & 0.96 & H \\
\hline
\end{tabular}

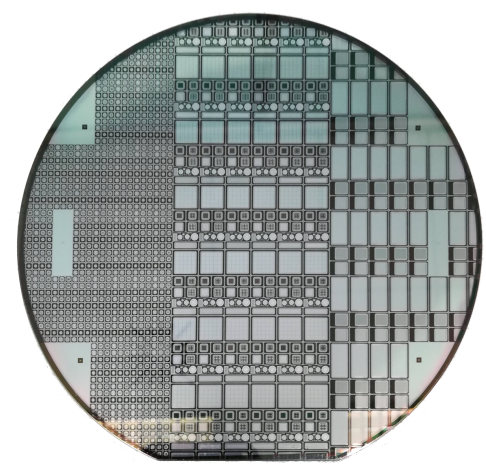

Figure 3: Summary of process split parameters in RSD1 batch (left) and final layout of the same production run at FBK (right).

The RSD1 layout included three lithographic shots, occupying different sectors of the wafer (see the right-hand side image of figure 3). The leftmost block includes detectors made by square matrices of pads with pitch from 50 to $300 \mu \mathrm{m}$ and different pad size. In the central block we implemented 500- $\mu \mathrm{m}$-pitch square matrices and some strip modules, while in the last block there are other strip-RSD devices for medical applications and two sensors expressly designed on the $64 \times 64$ channels $50 \times 50-\mu \mathrm{m}^{2}$-pitch RD53A chip [9]. 


\section{Detectors characterization}

The electrical tests performed on several RSD1 samples both in Trento and Torino revealed a very high uniformity in terms of implants within each wafer and among similar wafers. The breakdown voltage is generally high $(\sim 500 \mathrm{~V})$, it depends only on the gain layer dose and, as a consequence of the above mentioned uniformity, it resulted to be always the same for all the devices of a given wafer. For what concerns the transient properties, unirradiated RSD1 has been tested in the Silicon Laboratory at Università degli Studi di Torino and in the SSD Lab at CERN (EP-DT Department). Both campaigns confirmed that we succeeded in the challenging goal of achieving a very-fine-pitch level in Silicon detectors with internal gain and 100\% fill-factor.

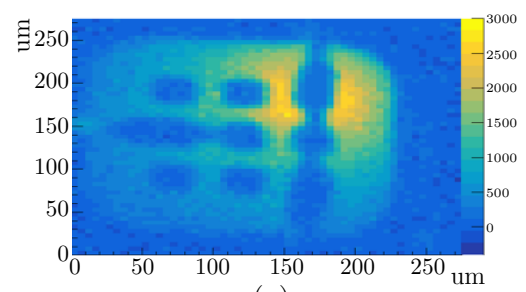

(a)

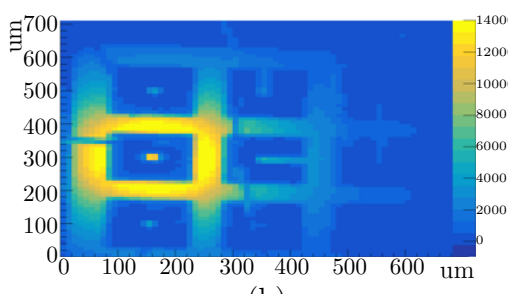

(b)

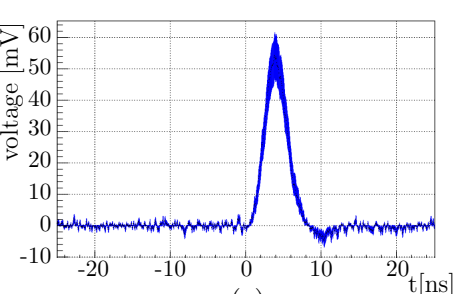

(c)

Figure 4: 2D maps of charges in a 50- (a) and 200- $\mu$ m-pitch (b) square matrix RSD1 sample, respectively from wafer 8 and 10, acquired with the TCT (Transient Current Technique) setup in Torino. Bipolar signal (c) coming from the sensor with pitch $200 \mu \mathrm{m}$. See more details in Ref. [8].

The 2D maps of charges obtained with a TCT (Transient Current Technique) [10] setup, and shown in panels (a) and (b) of figure 4, demonstrate that we have been able to design a correct readout scheme such that our RSD1 samples have an effective segmentation even with a pitch down to $50 \times 50 \mu \mathrm{m}^{2}$. Indeed, each AC-pad experiences a charge induction which surrounds the pad itself and extends up to the next neighboring pads. This produces a continuous and non-segmented coverage of the detector active area, at the basis of a $100 \%$ fill-factor pixelation scheme, proper of the RSD readout paradigm.

The second important aspect enabling the $4 \mathrm{D}$-tracking is the time resolution of the system. As already stated, the slew rate (and also the rise time) are directly related to the timing performance of a detector. Observing the signal reported in panel (c) of figure 4, what one may notice is the bipolar nature of the waveform, which is due to the capacitive induction in the first (positive) dominant lobe and determined by the discharge along the resistive layer for what concerns the small (negative) undershoot. All the signals acquired with the IR laser of our TCT setup in Torino have shown risetime values ranging from 500 to $700 \mathrm{ps,} \mathrm{which} \mathrm{is} \mathrm{perfectly} \mathrm{in} \mathrm{agreement} \mathrm{with} \mathrm{the}$ typical behavior of standard LGAD-based trackers. Furthermore, very preliminary measurements with a $\mathrm{Sr}^{90} \beta$-source indicated that a $300-\mu$ m-pitch square matrix RSD1 (i.e., a device not yet fully optimized for timing applications) can reach a time resolution not greater than $\sigma_{t} \sim 40 \mathrm{ps}$. But more investigations still have to be carried out. 


\section{Conclusions}

We simulated, designed, fabricated and tested a first run of RSD devices, called RSD1, that are Silicon detectors with internal gain and $100 \%$ fill-factor based on the resistive AC-coupled readout. The challenging goal of producing working sensors with very-fine pitch segmentation has been achieved, while the preliminary measurements of time resolution represent an encouraging feedback towards the use of the RSD paradigm for 4D particle tracking in present and future HEP experiments with high-luminosity environments.

\section{Acknowledgments}

We thank the RD50 and CMS collaborations at CERN and UC Santa Cruz for the scientific support, and also the technical staff at INFN Torino and FBK Trento. This work is funded by the INFN National Commission 5 under the Grant for Young Researchers 2017 (as in the announcement No.19105 and the related deliberation No.19567).

\section{References}

[1] M. Mandurrino (on behalf of the RD50 Collaboration at CERN), "Silicon Detectors for the LHC Phase-II Upgrade and Beyond. RD50 Status Report", Connecting the Dots / Workshop on Intelligent Trackers 2019 (CTD/WIT), València (Spain) 2-5 Apr 2019. Available online: arXiv:1910.06045.

[2] CMS Collaboration, "Technical proposal for a MIP timing detector in the CMS experiment Phase 2 upgrade". Available online: CERN-LHCC-2017-027; LHCC-P-009.

[3] G. Pellegrini et al., "Technology developments and first measurements of Low Gain Avalanche Detectors (LGAD) for high energy physics applications", Nucl. Instrum. Methods Phys. Res. A, vol. 765, pp. 12-16, 2014, DOI: 10.1016/j.nima.2014.06.008.

[4] N. Cartiglia et al., "Beam test results of a 16 ps timing system based on ultra-fast silicon detectors", Nucl. Instrum. Methods Phys. Res. A, vol. 850, pp. 83-88, 2017, DOI: 10.1016/j.nima.2017.01.021.

[5] H. F.-W. Sadrozinski, A. Seiden, N. Cartiglia, "4D tracking with ultra-fast silicon detectors”, Rep. Prog. Phys., vol. 81, p. 026101, 2018, DOI: 10.1088/1361-6633/aa94d3.

[6] M. Ferrero et al., "Radiation resistant LGAD design", Nucl. Instrum. Methods Phys. Res. A, vol. 919, pp. 16-26, 2019, DOI: 10.1016/j.nima.2018.11.121.

[7] J. Bendavid, "MIP Timing Detector for CMS Phase-II Upgrade", CMS MTD meeting, CERN, May 2018. Available online: https:/indico.cern.ch/event/726024/attachments/1641419/2627172/mtdSeminarCERN-May42018.pdf.

[8] M. Mandurrino et al., "Demonstration of 200, 100, and $50 \mu \mathrm{m}$ pitch Resistive AC-Coupled Silicon Detectors (RSD) with $100 \%$ fill-factor for 4D particle tracking”, preprint on IEEE Electron Device Lett. (2019), DOI: 10.1109/LED.2019.2943242.

[9] RD53 Collaboration, “The RD53A Integrated Circuit”. Available online: CERN-RD53-PUB-17-001.

[10] V. Eremin et al., "Development of transient current and charge techniques for the measurement of effective net concentration of ionized charges $\left(\mathrm{N}_{\mathrm{eff}}\right)$ in the space charge region of p-n junction detectors", Nucl. Instrum. Methods Phys. Res. A, vol. 372, no. 3, pp. 388-398, 1993, DOI: 10.1016/0168-9002(95)01295-8. 